\title{
Personalisierte Medizin. Medizinische, ethische, rechtliche und ökonomische Analysen
}

\author{
Jan Schildmann · Georg Marckmann · Jochen Vollmann
}

Online publiziert: 27. Juli 2013

(C) Springer-Verlag Berlin Heidelberg 2013

Die sogenannte ,personalisierte Medizin“ hat in den vergangenen Jahren große Hoffnungen bei Forschungsinstitutionen, Patienten, Leistungserbringern und auch in der Politik geweckt. In der wissenschaftlichen und gesellschaftlichen Diskussion werden mit dem Begriff unterschiedliche Interventionen bezeichnet. Gemeinsam ist den verschiedenen Ansätzen, dass auf der Grundlage von Biomarkern, zum Beispiel genetischer Merkmale, Subgruppen von Patienten identifiziert werden und Maßnahmen der Prävention, Diagnostik oder Therapie zielgerichteter durchgeführt werden sollen.

Mit Hilfe des Ansatzes der ,,personalisierten Medizin“, der sich dem Inhalt angemessen sprachlich am ehesten als „stratifizierende Medizin“ (von lateinisch stratum = Schicht und facere (in Zusammensetzung -ficere) = machen) fassen lässt, konnten in den vergangenen Jahren insbesondere im Bereich der Diagnostik und Therapie von einigen Krebserkrankungen Erfolge erzielt werden. Die Ergebnisse der biomedizinischen Forschung zeigen allerdings, dass eine zielgerichtete Behandlung derzeit nur für eine Minderheit von erkrankten Menschen zur Verfügung steht. Ein wesentlicher Grund ist die begrenzte Bedeutung einzelner genetischer Varianten für die Manifestation und den Verlauf von Erkrankungen. Für viele der sogenannten „Volkskrankheiten“ und auch für viele Tumorerkrankungen gilt vielmehr, dass unterschiedliche genetische Merkmale, umweltbedingte Faktoren sowie Wechselbeziehungen zwischen beiden für den Ausbruch und den Verlauf von Erkrankungen relevant sind. Eine weitere Schwierigkeit aus medizinischer Perspektive besteht im Mangel an qualitativ hochwertigen Testverfahren, mithilfe derer fundiert das Erkrankungsrisiko oder auch das Auftreten von erwünschten oder unerwünschten Wirkungen einer Therapie vorhergesagt werden könnten. Schließlich werden gegenwärtig mit Hilfe von sog. HochdurchsatzTechnologien zwar eine große Menge an Daten über mögliche Zusammenhänge zwischen

PD Dr. med. J. Schildmann, M.A. $(\bowtie)$ · Prof. Dr. med. Dr. phil. J. Vollmann Institut für Medizinische Ethik und Geschichte der Medizin, Ruhr-Universität Bochum, Malakowturm - Markstr. 258a, 44799 Bochum, Deutschland E-Mail: jan.schildmann@rub.de

Prof. Dr. med. G. Marckmann Institut für Ethik, Geschichte und Theorie der Medizin, Ludwig-Maximilians-Universität München, München, Deutschland 
genetischen Merkmalen und Erkrankungen generiert, aber gleichzeitig stehen die Expertise sowie die personellen und finanziellen Voraussetzungen für die Interpretation dieser Daten hinsichtlich ihrer Relevanz für die Gesundheitsversorgung nicht zur Verfügung.

Neben diesen und weiteren medizinisch-empirischen Problemstellungen ist die Umsetzung der ,personalisierten Medizin“ auch mit zahlreichen normativen Herausforderungen verbunden. Aus rechtlicher Perspektive werfen die Kombination von Diagnostika und Therapieverfahren (sogenannte companion diagnostics) sowie die Fokussierung auf kleine Patientensubgruppen im Kontext der „personalisierten Medizin“ die Frage auf, inwiefern Anpassungen bei der Regulierung von Zulassung und Kostenerstattung von Produkten der „personalisierten Medizin“ notwendig sind. Rechtliche und ethische Fragen stellen sich weiterhin hinsichtlich der informationellen Selbstbestimmung. Genetische Untersuchungen, die teilweise die Sequenzierung des gesamten Genoms eines Menschen umfassen, werfen nicht nur Fragen bezüglich des Schutzes von gesundheitsrelevanten Informationen gegenüber Dritten auf, sondern erfordern auch Güterabwägungen, wenn Informationen über genetische Merkmale nicht nur für die Gesundheit und das Leben der untersuchten Person, sondern auch für Familienmitglieder relevant sein könnten. Aus sozialethischer Perspektive stellt sich nicht zuletzt vor dem Hintergrund der bereits angesprochenen Unsicherheiten bezüglich der Bewertung des Nutzens von Biomarker basierten Interventionen die Frage, welche Ressourcen in der Forschung für die „personalisierte Medizin“ eingesetzt, beziehungsweise genauer, welcher konkrete Ansatz aus dem Spektrum stratifizierender Maßnahmen in Forschung, Prävention, Diagnostik und Therapie verfolgt werden soll. Diese Überlegungen umfassen auch Opportunitätskosten, etwa zuungunsten einer an psycho-sozialen Bedürfnissen orientierten patientenzentrierten Medizin. Auf der Ebene der Gesundheitsversorgung stellt sich weiterhin die Frage, wer von den Investitionen in die personalisierte Medizin und ihre Produkte profitieren wird. Der Zugang zu Leistungen der personalisierten Medizin, aber auch möglicher Diskriminierungen von Patientengruppen auf der Grundlage von genetischen Kennzeichen werden in diesem Zusammenhang diskutiert.

\section{Die Beiträge in der Kurzübersicht}

Das vorliegende Themenheft versammelt Forschungsergebnisse zu empirischen und normativen Aspekten der ,personalisierten Medizin“. Neben Arbeiten aus sechs Teilprojekten der beiden durch das Bundesministerium für Bildung und Forschung geförderten ELSA-Verbundforschungsprojekte ${ }^{1}$ konnten Wissenschaftler aus fünf weiteren deutschen Forschergruppen für die Mitarbeit gewonnen werden. Angesichts der Themenvielfalt und des begrenzten Umfangs des Themenheftes wurden alle Autoren gebeten, ihre Arbeiten möglichst kurz und pointiert darzustellen, um den Lesern einen Einblick in den Stand der Forschung zu Themen der ,personalisierten Medizin“ aus unterschiedlichen Disziplinen zu ermöglichen. Im Folgenden werden Schwerpunkte der einzelnen Arbeiten zur Orientierung für den Leser kurz vorgestellt.

\footnotetext{
${ }^{1}$ Informationen zu dem Verbundforschungsprojekt „Personalisierte Medizin in der Onkologie: ethische, medizinische, gesundheitsökonomische und rechtliche Aspekte" (Koordinator Prof. Dr. Dr. Jochen Vollmann) können unter http://www.ruhr-uni-bochum.de/malakow/bmbf-verb-pm/start.html abgerufen werden (zugegriffen: 18. Juni 2013). Informationen zum Verbundforschungsprojekt „Individualisierte Gesundheitsversorgung: Ethische, ökonomische rechtliche Implikationen für das deutsche Gesundheitswesen " (Koordinator Prof. Dr. Georg Marckmann, MPH) können unter http://www.igv-ethik.de/index.html abgerufen werden (zugegriffen: 18. Juni 2013).
} 
Die methodischen Herausforderungen auf dem Weg zu einer evidenzbasierten personalisierten Medizin stehen im Mittelpunkt des erstens Beitrags von Windeler und Lange. Die Autoren machen unter Verwendung von zahlreichen Beispielen aus der klinischen Forschung deutlich, dass Wechselwirkungen zwischen Biomarkern und den Effekten von zielgerichteten Interventionen nur im Rahmen prospektiv vergleichender Studien sicher identifiziert werden können.

Im Beitrag von Port et al. werden Ergebnisse einer systematischen Literaturanalyse zur Rolle eines Biomarkers für die Therapie der akuten myeloischen Leukämie (AML) untersucht. Die Untersuchung macht einerseits deutlich, wie Erkenntnisse zu Biomarkern die klinische Praxis bereits verändert haben. Andererseits ist die AML angesichts einer Vielzahl von inzwischen identifizierten genetischen Markern auch ein Beispiel für die Komplexität stratifizierender Ansätze bei der Behandlung von Tumorerkrankungen.

Die Implementierung von personalisierten Ansätzen in der Forschung und der klinischen Praxis zieht eine Vielzahl von Fragen hinsichtlich einer guten medizinischen Praxis nach sich. Ausgehend von auf Biomarker basierenden Forschungs- und Behandlungsansätzen am Nationalen Centrum für Tumorerkrankung in Heidelberg stellen Winkler et al. in ihrem Beitrag ausgewählte medizinethische und rechtliche Herausforderungen sowie Auszüge aus einem Verhaltenskodex zum Umgang mit genetischen Informationen vor.

Im ersten von zwei unter Anwendung von qualitativen sozialempirischen Methoden erstellten Beiträgen zu ethischen Aspekten der personalisierten Medizin berichten Wäscher et al. über Ergebnisse einer Interviewstudie mit onkologisch forschenden und in der klinischen Praxis tätigen Ärzten. Im Zentrum der empirisch-ethischen Analyse stehen die Wahrnehmungen und Bewertungen der Befragten hinsichtlich der Auswirkungen auf die Versorgung von an Krebs erkrankten Menschen.

In der zweiten empirisch-ethischen Untersuchung berichten Wöhlke et al. über Ergebnisse aus einer laufenden qualitativen Studie, in der die Perspektiven von klinischen Forschern und Patienten bezüglich eines Forschungsvorhabens zu einem genetisch gestützten Ansatz zur Therapie des Rektumkarzinoms exploriert werden. Im Mittelpunkt steht die Identifizierung ethisch relevanter Konfliktfelder auf der Grundlage der Perspektiven der unterschiedlichen Stakeholder.

Im Mittelpunkt des ethisch-konzeptionellen Beitrags von Schleidgen und Marckmann steht die Präzisierung des Gegenstandsbereichs einer ,individualisierten Medizin“ sowie die Differenzierung der damit verbundenen ethischen Herausforderungen. Im Anschluss an einen Definitionsvorschlag für den Begriff der ,individualisierten Medizin“ präsentieren die Autoren eine Sechs-Felder-Matrix zur Kategorisierung individual- und sozialethischer Fragestellungen in den Bereichen Forschung, Prävention und Therapie.

Im Beitrag von Vollmann werden die fragwürdigen Erwartungen, die mit dem Begriff „personalisierte Medizin“ verbunden sind, und die Folgen einer einseitigen Prioritätensetzung zugunsten dieses Ansatzes in der Medizin analysiert. Nach einer Kritik des Begriffs „personalisierte Medizin“ angesichts der Diskrepanz zwischen dem ausschließlich biologisch orientierten Konzept der stratifizierenden Medizin und dem normativen PersonenKonzept werden normative und praktische Folgen einer überwiegend forschungs- und industriegesteuerten ,personalisierten Medizin“ für das öffentliche Gesundheitssystem diskutiert.

In ihrer logischen und normativen Analyse untersuchen Langanke et al. die in der Diskussion häufig vorausgesetzte Verbindung von ,,personalisierter Medizin“ und gesundheitsbezogener Eigenverantwortung. Die Autoren argumentieren, dass auch bei verlässlicher Vorhersage anlagebedingter und zugleich lebensstilabhängiger Erkrankungsrisiken logisch nicht auf eine Forderung nach Sanktionierung von gesundheitsgefährdenden Lebensstilen 
geschlossen werden kann. Vielmehr beruhe die Forderung nach gesundheitlicher Eigenverantwortung auf weiteren normativen Prinzipien, die ihrerseits begründet werden müssten.

Einen methodisch orientierten Beitrag zu ethischen Aspekten der ,,personalisierten Medizin“ leisten Braun et al. Die Autoren unterscheiden anhand des Modells einer „First-und Second-Wave-Bioethics“ eine „,erste Welle“, im Verlauf derer es zu ersten kritischen Rückfragen an die personalisierte Medizin kommt, und eine „zweite Welle“, im Verlauf derer die formulierten Anfragen einer genaueren ethischen Metabeobachtung unterzogen werden. In ihrer eigenen (sozial-)ethischen Analyse fokussieren die Autoren auf das Konzept der „genetischen Risikoperson“.

Rechtliche Fragen einer möglichen „Orphanisierung“ im Kontext der „personalisierten Medizin“ stehen im Mittelpunkt des Beitrags von Gottwald und Huster. Mit Blick auf die derzeit geltende Ausnahme einer Nachweispflicht für einen Zusatznutzen bei sogenannten Orphan Drugs, also Arzneimittel für seltene Erkrankungen, werden Implikationen einer „Orphanisierung“ durch die Entwicklung von Substanzen für kleine Patientengruppen im Kontext der „personalisierten Medizin“ untersucht.

Die beiden abschließenden Beiträge des Themenhefts widmen sich gesundheitsökonomischen Fragestellungen im Kontext der ,personalisierten Medizin“. Im ersten Beitrag von Sickinger et al. steht die Frage der Übertragbarkeit methodischer Ansätze der Gesundheitsökonomie auf die ,personalisierte Medizin“ im Mittelpunkt. Die Autoren unterscheiden dabei eine ,personalisierte Medizin“, die sich auf physiologische Unterschiede bezieht, von einer Strategie, die auf die individuellen Präferenzen der Beteiligten abzielt. Für beide Ansätze bestehen Möglichkeiten der gesundheitsökonomischen Evaluation, die von den Autoren vorgestellt und diskutiert werden.

Im zweiten gesundheitsökonomischen Beitrag des Themenhefts untersuchen Noweski et al. die Rahmenbedingungen für Arzneimittelzulassungen und den Aufnahmeprozess für die Kostenerstattung unter Berücksichtigung möglicher förderlicher beziehungsweise behindernder Faktoren. Die Autoren stellen fest, dass keine unzumutbaren Hürden für die Hersteller bestehen, die eine Zurückhaltung bei der Entwicklung von Produkten erklären würden.

Die Herausgeber hoffen, dass dieses ein breites Spektrum an Inhalten, Methoden und Disziplinen versammelnde Themenheft zur ,,personalisierten Medizin“ den Lesern unterschiedlicher Disziplinen und professioneller Kontexte einen guten Einblick in den Forschungsstand geben kann. Nach Einschätzung der Herausgeber bietet das Themenheft in der vorgelegten Form auch einen guten Ausgangspunkt für zukünftige disziplinspezifische, wie auch interdisziplinäre Forschungsvorhaben zur Bearbeitung ausgewählter normativer und empirischer Fragestellungen. Nicht zuletzt angesichts der Dynamik der Entwicklung und der gegenwärtigen Prioritätensetzung in Wissenschaft und Politik einerseits und den Unsicherheiten hinsichtlich der Implikationen der ,personalisierten Medizin“ für die Gesundheitsversorgung andererseits erscheint eine weitergehende konstruktiv-kritische Begleitforschung nicht nur wünschenswert sondern erforderlich.

Wir möchten uns abschließend bei allen Autorinnen und Autoren für die gute Zusammenarbeit bedanken. Weiterhin danken wir den externen Reviewern für die Begutachtung von Beiträgen. Wir danken dem Bundesministerium für Forschung und Bildung für die Förderung dieser über den normalen Umfang eines Themenheftes deutlich hinausgehenden Publikation (FK: 01GP1001A). Frau Verena Sandow und Frau Andrea Kähling danken wir für die sorgfältige redaktionelle Bearbeitung der Beiträge. 